Nonlinear Analysis: Real World Applications, Vol. 10, № 2, 601-610 (2009)

\title{
Application of a modified He's homotopy perturbation method to obtain higher-order approximations to a nonlinear oscillator with discontinuities
}

\section{A. Beléndez, C. Pascual, M. Ortuño, T. Beléndez and S. Gallego}

Departamento de Física, Ingeniería de Sistemas y Teoría de la Señal. Universidad de Alicante. Apartado 99. E-03080 Alicante. SPAIN

E-mail: a.belendez@ua.es

Corresponding author: A. Beléndez

Phone: +34-96-5903651

Fax: +34-96-5909750 


\begin{abstract}
A modified He's homotopy perturbation method is used to calculate the periodic solutions of a nonlinear oscillator with discontinuities for which the elastic force term is proportional to $\operatorname{sgn}(x)$. The He's homotopy perturbation method is modified by truncating the infinite series corresponding to the first-order approximate solution before introducing this solution in the second order linear differential equation. We find this modified homotopy perturbation method works very well for the whole range of initial amplitudes, and the excellent agreement of the approximate frequencies and periodic solutions with the exact ones has been demonstrated and discussed. Only one iteration leads to high accuracy of the solutions with a maximal relative error for the approximate period of less than $1.6 \%$ for all values of oscillation amplitude, while this relative error is $0.65 \%$ for the second iteration and $0.24 \%$ when the third-order approximation is considered. Comparison of the result obtained using this method with the exact ones reveals that this modified method is very effective and convenient.
\end{abstract}

Keywords: Nonlinear oscillator; Approximate solutions; Homotopy perturbation method. 


\section{Introduction}

The study of nonlinear problems is of crucial importance in all areas of Physics and Engineering, as well as in other disciplines. It is very difficult to solve nonlinear problems and, in general, it is often more difficult to get an analytic approximation than a numerical one to a given nonlinear problem. There are several methods used to find approximate solutions to nonlinear problems, such as perturbation techniques [1-7], harmonic balance based methods [8-12] or other techniques [13-18]. An excellent review on some asymptotic methods for strongly nonlinear equations can be found in detail in references [19] and [20].

In the present paper we obtain higher-order analytical approximations to the periodic solutions to a nonlinear oscillator with discontinuities for which the elastic restoring force is an antisymmetric and constant force. To do this, we apply a modified He's homotopy perturbation method [21]. This type of oscillator has been analyzed by Liu [22] and by Özis and Yildirim [23] applying the first-order homotopy perturbation method. They also make the comparison of two distinct adaptations of the first-order homotopy perturbation method for determining frequencyamplitude relation of the nonlinear oscillator with discontinuities. However, higher-order analytical approximate solutions have not been obtained for this oscillator using He's homotopy perturbation method.

Applying the standard He's homotopy perturbation method [24-40] to this nonlinear oscillator with discontinuities, an infinite series is obtained for the first analytical approximate solution and this series must be introduced in the linear differential equation to obtain the secondorder approximate solution. However, since it is difficult to work with an infinite series, we truncate this series before solving the subsequent linear differential equation considering only two harmonics for the second order approximation. As we can see, the results presented in this paper reveal that the method is very effective and convenient for conservative nonlinear oscillators for which the restoring force has a non-polynomial form.

\section{Solution procedure}

The antisymmetric, piecewise constant force oscillator, is governed by the following differential equation

$$
\frac{\mathrm{d}^{2} x}{\mathrm{~d} t^{2}}+\operatorname{sgn}(x)=0
$$


with initial conditions

$$
x(0)=A \text { and } \quad \frac{\mathrm{d} x}{\mathrm{~d} t}(0)=0
$$

and $\operatorname{sgn}(x)$ is defined as

$$
\begin{array}{ll}
\operatorname{sgn}(x)=-1, & x<0 \\
\operatorname{sgn}(x)=+1, & x>0
\end{array}
$$

Equation (1) models the motion of a punctual ball rolling in a "V" shape trough in a constant gravitational field. The arms of the "V" make equal angles with horizontal plane and the origin of the (horizontal) $x$ coordinate is taken to the point of interaction of the two arms [1]. In a suitable set of units, the equation of motion can be written as Eq. (1).

All the solutions to Eq. (1) are periodic. We denote the angular frequency of these oscillations by $\omega$ and note that one of our major tasks is to determine $\omega(A)$, i.e., the functional behaviour of $\omega$ as a function of the initial amplitude $A$.

There exist no small parameter in Eq. (1), so the traditional perturbation methods can not be applied directly, moreover the equation involves discontinuity. Due to the fact that the homotopy perturbation method requires neither a small parameter nor a linear term in a differential equation, we can approximately solve Eq. (1) using the homotopy perturbation method. In this method, an artificial perturbation equation is constructing by embedding an artificial parameter $p \in[0,1]$, which is used as expanding parameter. This technique yields a very rapid convergence of the solution series; in most cases, only one iteration leads to high accuracy of the solution. This method provides an effective and convenient mathematical tool for nonlinear differential equations [20].

Equation (1) can be re-written in the form

$$
\frac{\mathrm{d}^{2} x}{\mathrm{~d} t^{2}}+x=x-\operatorname{sgn}(x)
$$


For Eq. (4) we can establish the following homotopy

$$
\frac{\mathrm{d}^{2} x}{\mathrm{~d} t^{2}}+x=p[x-\operatorname{sgn}(x)]
$$

where $p$ is the homotopy parameter. When $p=0$, Eq. (5) becomes a linear differential equation for which an exact solution can be calculated; for $p=1$, Eq. (5) then becomes the original problem. Now the homotopy parameter $p$ is used to expand the solution $x(t)$ and the square of the unknown angular frequency $\omega$ as follows

$$
\begin{gathered}
x(t)=x_{0}(t)+p x_{1}(t)+p^{2} x_{2}(t)+\ldots \\
1=\omega^{2}-p \alpha_{1}-p^{2} \alpha_{2}-\ldots
\end{gathered}
$$

where $\alpha_{i}(i=1,2, \ldots)$ are to be determined.

Substituting Eqs. (6) and (7) into Eq. (5)

$$
\begin{aligned}
& \left(x_{0}^{\prime \prime}+p x_{1}^{\prime \prime}+p^{2} x_{2}^{\prime \prime}+\ldots\right)+\left(\omega^{2}-p \alpha_{1}-p^{2} \alpha_{2}-\ldots\right)\left(x_{0}+p x_{1}+p^{2} x_{2}+\ldots\right)= \\
& =p\left[\left(x_{0}+p x_{1}+p^{2} x_{2}+\ldots\right)-\operatorname{sgn}\left(x_{0}+p x_{1}+p^{2} x_{2}+\ldots\right)\right]
\end{aligned}
$$

and collecting the terms of the same power of $p$, we obtain a series of linear equations, of which we write only the first four

$$
\begin{gathered}
x_{0}^{\prime \prime}+\omega^{2} x_{0}=0, \quad x_{0}(0)=A, \quad x_{0}^{\prime}(0)=0 \\
x_{1}^{\prime \prime}+\omega^{2} x_{1}=\left(1+\alpha_{1}\right) x_{0}-\operatorname{sgn}\left(x_{0}\right), \quad x_{1}(0)=x_{1}^{\prime}(0)=0 \\
x_{2}^{\prime \prime}+\omega^{2} x_{2}=\alpha_{2} x_{0}+\left(1+\alpha_{1}\right) x_{1}, \quad x_{2}(0)=x_{2}^{\prime}(0)=0
\end{gathered}
$$




$$
x_{3}^{\prime \prime}+\omega^{2} x_{3}=\alpha_{3} x_{0}+\alpha_{2} x_{1}+\left(1+\alpha_{1}\right) x_{2}, \quad x_{3}(0)=x_{3}^{\prime}(0)=0
$$

In Eqs. (9)-(12) we have taken into account the following expression

$$
f(x)=f\left(x_{0}+p x_{1}+p^{2} x_{2}+\ldots\right)=f\left(x_{0}\right)+p x_{1} f^{\prime}\left(x_{0}\right)+p^{2}\left[x_{2} f^{\prime}\left(x_{0}\right)+\frac{1}{2} x_{1}^{2} f^{\prime \prime}\left(x_{0}\right)\right]+O\left(p^{3}\right)
$$

where $f^{\prime}(x)=\mathrm{d} f(x) / \mathrm{d} x$ and

$$
\frac{\mathrm{d} \operatorname{sgn}(x)}{\mathrm{d} x}=\frac{\mathrm{d}^{2} \operatorname{sgn}(x)}{\mathrm{d} x^{2}}=\ldots=0 \text { for } x \neq 0
$$

and

$$
\operatorname{sgn}\left(x_{0}+p x_{1}+p^{2} x_{2}+\ldots\right)=\operatorname{sgn}\left(x_{0}\right)
$$

The solution of Eq. (9) is

$$
x_{0}(t)=A \cos \omega t
$$

Substitution of this result into the right side of Eq. (10) gives

$$
x_{1}^{\prime \prime}+\omega^{2} x_{1}=\left(1+\alpha_{1}\right) A \cos \omega t-\operatorname{sgn}(A \cos \omega t)
$$

It is possible to do the following Fourier series expansion

$$
\operatorname{sgn}(\cos \omega t)=\sum_{n=0}^{\infty} a_{2 n+1} \cos [(2 n+1) \omega t]=a_{1} \cos \omega t+a_{3} \cos 3 \omega t+\ldots
$$

where

$$
a_{2 n+1}=\frac{4}{\pi} \int_{0}^{\pi / 2} \operatorname{sgn}(A \cos \theta) \cos [(2 n+1) \theta] \mathrm{d} \theta
$$


The first term of the expansion in Eq. (16) is given by

$$
a_{1}=\frac{4}{\pi} \int_{0}^{\pi / 2} \operatorname{sgn}(A \cos \theta) \cos \theta \mathrm{d} \theta=\frac{4}{\pi}
$$

Substituting Eq. (16) into Eq. (15), we have

$$
x_{1}^{\prime \prime}+\omega^{2} x_{1}=\left[\left(1+\alpha_{1}\right) A-a_{1}\right] \cos \omega t-\sum_{n=1}^{\infty} a_{2 n+1} \cos [(2 n+1) \omega t]
$$

No secular terms in $x_{1}(t)$ requires eliminating contributions proportional to $\cos \omega t$ on the right-hand side of Eq. (19)

$$
\left(1+\alpha_{1}\right) A-a_{1}=0
$$

From this equation we can easily find that the solution $\alpha_{1}$ is

$$
\alpha_{1}=-1+\frac{a_{1}}{A}
$$

where $a_{1}=4 / \pi$ (Eq. (18)). From Eqs. (7), (18) and (21), writing $p=1$, we can easily find that the first order approximate frequency is

$$
\omega_{1}(A)=\sqrt{\frac{a_{1}}{A}}=\frac{2}{\sqrt{\pi A}}=\frac{1.128379}{\sqrt{A}}
$$

and the first-order approximate period $T_{1}(A)=2 \pi / \omega_{1}(A)$ is given as follows

$$
T_{1}(A)=\pi \sqrt{\pi A}=5.568328 \sqrt{A}
$$


which agrees exactly with Liu's solution obtained by He's modified Lindsted-Poincaré method [22] and with low-order harmonic balance method [41].

Now in order to obtain the correction term $x_{1}$ for the periodic solution $x_{0}$ we consider the following procedure. Taking into account Eqs. (19) and (21), we re-write Eq. (19) in the form

$$
x_{1}^{\prime \prime}+\omega^{2} x_{1}=-\sum_{n=1}^{\infty} a_{2 n+1} \cos [(2 n+1) \omega t]
$$

with initial conditions $x_{1}(0)=0$ and $x_{1}^{\prime}(0)=0$. The periodic solution to Eq. (24) can be written

$$
x_{1}(t)=\sum_{n=0}^{\infty} b_{2 n+1} \cos [(2 n+1) \omega t]
$$

Substituting Eq. (25) into Eq. (24) gives

$$
-\omega^{2} \sum_{n=0}^{\infty} 4 n(n+1) b_{2 n+1} \cos [(2 n+1) \omega t]=-\sum_{n=1}^{\infty} a_{2 n+1} \cos [(2 n+1) \omega t]
$$

and then we can write the following expression for the coefficients $b_{2 n+1}$

$$
b_{2 n+1}=\frac{a_{2 n+1}}{4 n(n+1) \omega^{2}}
$$

for $n \geq 1$. Taking into account that $x_{1}(0)=0$, Eq. (25) gives

$$
b_{1}=-\sum_{n=1}^{\infty} b_{2 n+1}
$$

To determine the second-order approximate solution it is necessary to substitute Eq. (25) into Eq. (11). Then secular terms are eliminated and parameter $\alpha_{2}$ can be calculated. However, it 
is difficult to solve the new differential equation because, as $x_{1}(t)$ has a infinite number of harmonics (see Eq. (25)). At this moment we consider a modification in He's homotopy perturbation method to simplify the solution procedure. $x_{1}(t)$ has an infinite number of harmonics, however we can truncate the series expansion at Eq. (25) and write an approximate equation $x_{1}^{(N)}(t)$ in the form

$$
x_{1}^{(N)}=\sum_{n=0}^{N} b_{2 n+1} \cos [(2 n+1) \omega t]
$$

where

$$
b_{1}^{(N)}=-\sum_{n=1}^{N} b_{2 n+1}
$$

Equation (29) has only a finite number of harmonics. Comparing Eqs. (25) and (29), and Eqs. (28) and (30), it follows that

$$
\lim _{N \rightarrow \infty} x_{1}^{(N)}(t)=x_{1}(t)
$$

and

$$
\lim _{N \rightarrow \infty} b_{1}^{(N)}=b_{1}
$$

In the simplest case we consider $N=1(n=0,1)$ in Eq. (29) and Eq. (30), we obtain

$$
x_{1}^{(1)}(t)=b_{3}(\cos 3 \omega t-\cos \omega t)
$$

which has a similar form than the second order approximate solution considered in harmonic balance methods.

From Eq. (27) the following expression for the coefficient $b_{3}$ is obtained

$$
b_{3}=\frac{a_{3}}{8 \omega^{2}}
$$


where

$$
a_{3}=\frac{4}{\pi} \int_{0}^{\pi / 2} \operatorname{sgn}(A \cos \theta) \cos 3 \theta \mathrm{d} \theta=-\frac{4}{3 \pi}
$$

Therefore, the first approximation to the periodic solution is given by

$x_{a 1}(t)=x_{0}(t)+x_{1}^{(1)}(t)=\frac{25}{24} A \cos \left(\omega_{1} t\right)-\frac{1}{24} A \cos \left(3 \omega_{1} t\right)=1.04167 A \cos \left(\omega_{1} t\right)-0.04167 A \cos \left(3 \omega_{1} t\right)$

where $\omega_{1}(A)$ is given by Eq. (22).

Substitution of Eq. (29) (with $N=1$ ) into Eq. (11) gives the following equation for gives the following equation for $x_{2}(t)$

$$
x_{2}^{\prime \prime}+\omega^{2} x_{2}=\alpha_{2} x_{0}+\left(1+\alpha_{1}\right) x_{1}^{(1)}
$$

and taking into account Eqs. (14), (21), (33) and (34), Eq. (37) becomes

$$
x_{2}^{\prime \prime}+\omega^{2} x_{2}=\alpha_{2} A \cos \omega t+\frac{a_{1} a_{3}}{8 A \omega^{2}}(\cos 3 \omega t-\cos \omega t)
$$

The secular term in the solution for $x_{2}(t)$ can be eliminated if

$$
\alpha_{2} A-\frac{a_{1} a_{3}}{8 A \omega^{2}}=0
$$

which can be solved for $\alpha_{2}$, that is

$$
\alpha_{2}=\frac{a_{1} a_{3}}{8 A^{2} \omega^{2}}
$$


From Eqs. (7), (21) and (40), and taking $p=1$, one can easily obtain the following expression for the second-order approximate frequency is

$$
\omega_{2}(A)=\sqrt{\frac{a_{1}}{2 A}+\frac{1}{2 A} \sqrt{\frac{2 a_{1}^{2}+a_{1} a_{3}}{2}}}=\sqrt{\frac{6+\sqrt{30}}{3 \pi A}}=\frac{1.103527}{\sqrt{A}}
$$

and the second-order approximate period $T_{2}(A)=2 \pi / \omega_{2}(A)$ is given as follows

$$
T_{2}(A)=5.693731 \sqrt{A}
$$

With the requirement of Eq. (39), we can re-write Eq. (38) in the form

$$
x_{2}^{\prime \prime}+\omega^{2} x_{2}=\frac{a_{1} a_{3}}{8 A \omega^{2}} \cos 3 \omega t
$$

with initial conditions $x_{2}(0)=0$ and $x_{2}^{\prime}(0)=0$. The solution of this equation is

$$
x_{2}(t)=\frac{a_{1} a_{3}}{8 A \omega^{4}}(\cos \omega t-\cos 3 \omega t)
$$

which has only a finite number of harmonics.

Therefore, the second approximation to the periodic solution is given by

$$
x_{a 2}(t)=x_{0}(t)+x_{1}^{(1)}(t)+x_{2}(t)=1.03787 A \cos \left(\omega_{2} t\right)-0.03787 A \cos \left(3 \omega_{2} t\right)
$$

where $\omega_{2}(A)$ is given by Eq. (41).

If Eqs. (14), (21), (33), (40) and (44) are substituted into Eq. (12), then the following equation is obtained for $x_{3}(t)$

$$
x_{3}^{\prime \prime}+\omega^{2} x_{3}=\alpha_{3} A \cos \omega t+\frac{a_{1} a_{3}^{2}}{64 A^{2} \omega^{4}}(\cos 3 \omega t-\cos \omega t)-\frac{a_{1}^{2} a_{3}}{64 A^{2} \omega^{4}}(\cos 3 \omega t-\cos \omega t)
$$


Following the same procedure used for calculating $x_{1}(t)$ and $x_{2}(t)$, the secular term in the solution for $x_{3}(t)$ can be eliminated if

$$
\alpha_{3} A-\frac{a_{1} a_{3}^{2}}{64 A^{2} \omega^{4}}+\frac{a_{1}^{2} a_{3}}{64 A^{2} \omega^{4}}=0
$$

which can be solved for $\alpha_{3}$, that is

$$
\alpha_{3}=\frac{a_{1} a_{3}^{2}-a_{1}^{2} a_{3}}{64 A^{3} \omega^{4}}
$$

Considering Eqs. (7), (21), (40) and (48), and imposing $p=1$, give for the third-order approximate frequency the following value

$$
\omega_{3}(A)=\frac{1}{\sqrt{3 \pi A}} \sqrt{4+7 \frac{2^{2 / 3}}{(29+\sqrt{155})^{1 / 3}}+[2(29+\sqrt{155})]^{1 / 3}}=\frac{1.108030}{\sqrt{A}}
$$

and the third-order approximate period $T_{3}(A)=2 \pi / \omega_{3}(A)$ is given as follows

$$
T_{3}(A)=5.670590 \sqrt{A}
$$

With the requirement of Eq. (48), we can re-write Eq. (46) in the form

$$
x_{3}^{\prime \prime}+\omega^{2} x_{3}=\frac{a_{1} a_{3}^{2}-a_{1}^{2} a_{3}}{64 A^{2} \omega^{4}} \cos 3 \omega t
$$

with initial conditions $x_{3}(0)=0$ and $x_{3}^{\prime}(0)=0$. The solution of this equation is

$$
x_{3}(t)=\frac{a_{1} a_{3}^{2}-a_{1}^{2} a_{3}}{512 A^{2} \omega^{6}}(\cos \omega t-\cos 3 \omega t)
$$


Therefore, the third approximation to the periodic solution is given by

$$
x_{a 3}(t)=x_{0}(t)+x_{1}^{(1)}(t)+x_{2}(t)+x_{3}(t)=1.03858 A \cos \left(\omega_{3} t\right)-0.03858 A \cos \left(3 \omega_{3} t\right)
$$

where $\omega_{3}(A)$ is given by Eq. (49).

\section{Results and discussion}

We illustrate the accuracy of the modified approach by comparing the approximate solutions previously obtained with the exact solution.

For this nonlinear problem, the exact period and periodic solution are [41]

$$
\begin{array}{cc}
T_{e}(A)=4 \sqrt{2 A}=5.656854 \sqrt{A} & 0 \leq t \leq \frac{T_{e}}{4} \\
x_{e}(t)=-\frac{t^{2}}{2}+A, & \frac{T_{e}}{4}<t \leq \frac{3 T_{e}}{4} \\
x_{e}(t)=\frac{t^{2}}{2}-2 \sqrt{2 A} t+3 A, & \frac{3 T_{e}}{4}<t \leq T_{e} \\
x_{e}(t)=-\frac{t^{2}}{2}+4 \sqrt{2 A} t-15 A, &
\end{array}
$$

The period values and their relative errors $(R E)$ obtained in this paper applying a modified He's homotopy perturbation method to this nonlinear oscillator with discontinuities are the following

$$
\begin{array}{cc}
T_{1}(A)=5.568328 \sqrt{A} & R E=1.6 \% \\
T_{2}(A)=5.693731 \sqrt{A} & R E=0.65 \% \\
T_{3}(A)=5.670590 \sqrt{A} & R E=0.24 \%
\end{array}
$$


where the percentage errors $(R E)$ were calculated using the following equation

$$
R E(\%)=100\left|\frac{T_{j}(A)-T_{e}(A)}{T_{e}(A)}\right| \quad j=1,2,3
$$

It can be observed that these equations provide excellent approximations to the exact period regardless of the oscillation amplitude $A$. It is also clear that at the third approximation order, the accuracy of the result obtained in this paper is very good.

The normalized exact periodic solution $x_{e} / A$ in Eq. (55) and the first-, second- and thirdorder approximate periodic solutions, $x_{a} / A$ in Eqs. (36), (45) and (53), are plotted in Figure 1, 2 and 3 , respectively. In these figures parameter $h$ is defined as follows

$$
h=T_{e}(A) t=\frac{2 \pi t}{\omega_{e}(A)}
$$

It can be observed that the third-order approximate solution provides excellent approximation to the exact periodic solution.

An easy and direct calculation gives the following series representation for the exact solution $x_{e}(t)$ (Eq. (55))

$$
x_{e}(t)=\frac{32 A}{\pi^{3}} \sum_{n=0}^{\infty} \frac{(-1)^{n}}{(2 n+1)^{3}} \cos \left[(2 n+1) \omega_{e} t\right]
$$

where

$$
\omega_{e}(A)=\frac{2 \pi}{T_{e}(A)}=\frac{\pi}{2 \sqrt{2 A}}=\frac{1.110721}{\sqrt{A}}
$$

Also the condition $x_{e}(0)=A$ gives the result

$$
\sum_{n=0}^{\infty} \frac{(-1)^{n}}{(2 n+1)^{3}}=\frac{\pi^{3}}{32}
$$


The first terms of the Fourier expansion in Eq. (61) are

$x_{e}(t)=1.03205 A \cos \omega_{e} t-0.03822 A \cos 3 \omega_{e} t+0.008256 A \cos 5 \omega_{e} t-0.003009 A \cos 7 \omega_{e} t+\ldots$

If we compare the approximate solutions for the first, the second and the third analytical approximations with the Fourier expansion of the exact solution (Eq. (64)), we can see that $x_{a 3}(t)$ is the best approximation. If we compare $x_{a 3}(t)$ (Eq. (52)) with the first two terms of the Fourier expansion of $x_{e}(t)$ (Eq. (64)) we can see that the relative errors for these terms are $0.63 \%$ and $0.93 \%$, respectively. If we compare $x_{a 2}(t)$ (Eq. (45)) with the first two terms of the Fourier expansion of $x_{e}(t)$ (Eq. (64)) we can see that the relative errors for these terms are $0.56 \%$ and $0.93 \%$, respectively. As we can see, these errors are similar to those obtained for $x_{a 3}(t)$. However $x_{a 3}(t)$ is a better approximation than $x_{a 3}(t)$ because the approximate period obtained using the third-order homotopy perturbation method, $T_{3}(A)$ (Eq. (58)), is better than the period obtained by means of the second-order homotopy perturbation method, $T_{2}(A)$ (Eq. (57)). Finally, if we compare $x_{a 1}(t)$ (Eq. (36)) with the first two terms of the Fourier expansion of $x_{e}(t)$ (Eq. (64)) we can see that the relative errors for these terms are $0.93 \%$ and $9.0 \%$, respectively.

\section{Conclusions}

The homotopy perturbation method has been used to obtain three approximate frequencies for a conservative antisymmetric, constant force nonlinear oscillator for which the elastic force term is proportional to $\operatorname{sgn}(x)$. Excellent agreement between approximate periods and the exact one has been demonstrated and discussed, and the discrepancy of the third-order approximate period with respect to the exact one is as low as $0.24 \%$. Finally, we can see that the method considered here is very simple in its principle and we think that the method has great potential and can be applied to other strongly nonlinear oscillators with non-polynomial terms.

\section{Acknowledgements}

This work was supported by the "Ministerio de Educación y Ciencia", Spain, under project FIS2005-05881-C02-02 and the "Generalitat Valenciana", Spain, under project ACOMP/ 2007/020. 


\section{References}

[1] R. E. Mickens, Oscillations in Planar Dynamics Systems (World Scientific, Singapore 1996).

[2] J. H. He, "Modified Lindstedt-Poincare methods for some non-linear oscillations. Part III: double series expansion”, Int. J. Non-linear Sci. Numer. Simulation 2, 317-320 (2001).

[3] J. H. He, "Modified Lindstedt-Poincare methods for some non-linear oscillations. Part I: expansion of a constant”, Int. J. Non-linear Mech. 37, 309-314 (2002).

[4] P. Amore and F. M. Fernández, "Exact and approximate expressions for the period of anharmonic oscillators", Eur. J. Phys. 26, 589-601 (2005).

[5] P. Amore, A. Raya and F. M. Fernández, "Alternative perturbation approaches in classical mechanics”, Eur. J. Phys. 26, 1057-1063 (2005).

[6] J. H. He, "A new perturbation technique which is also valid for large parameters", J. Sound Vib. 229, 1257-1263 (2000).

[7] T. Özis and A. Yildirim, "Determination of periodic solution for a $u^{1 / 3}$ force by He's modified Lindstedt-Poincaré method"; J. Sound Vib. 301, 415-419 (2007).

[8] H. P. W. Gottlieb, "Harmonic balance approach to limit cycles for nonlinear jerk equations", J. Sound Vib. 297, 243-250 (2006).

[9] C. W. Lim, B. S. Wu and W. P. Sun, "Higher accuracy analytical approximations to the Duffing-harmonic oscillator", J. Sound Vib. 296, 1039-1045 (2006).

[10] A. Beléndez, A. Hernández, A. Márquez, T. Beléndez and C. Neipp, "Analytical approximations for the period of a simple pendulum”, Eur. J. Phys. 27, 539-551 (2006).

[11] G. R. Itovich and J. L. Moiola, "On period doubling bifurcations of cycles and the harmonic balance method", Chaos, Solitons \& Fractals 27, 647-665 (2005).

[12] A. Beléndez, A. Hernández, T. Beléndez, M. L. Álvarez, S. Gallego, M. Ortuño and C. Neipp, "Application of the harmonic balance method to a nonlinear oscillator typified by a mass attached to a stretched wire", J. Sound Vib. 302, 1018-1029 (2007).

[13] J. H. He, "Application of parameter-expanding method to strongly nonlinear oscillators", Int. J. Non-linear Sci. Numer. Simulation 8 (1), 121-124 (2007). 
[14] A. Beléndez, A. Hernández, T. Beléndez, A. Márquez and C. Neipp, “An improved 'heuristic' approximation for the period of a nonlinear pendulum: linear analysis of a classical nonlinear problem", Int. J. Non-linear Sci. Numer. Simulation 8 (3), 329-334 (2007).

[15] L. Geng and X. C. Cai, "He's formulation for nonlinear oscillators", Eur. J. Phys. Eur. J. Phys. 28, 923-931 (2007).

[16] J. H. He and X. H. Wu, "Construction of solitary solution and compact on-like solution by variational iteration method", Chaos, Solitons \& Fractals 29, 108-113 (2006).

[17] M. Dehghan and M. Tatari, "Te use of He's variational iteration method for solving multipoint boundary value problems", Phys. Scr. 72, 672-676 (2007).

[18] J. H. He, "Variational approach for nonlinear oscillators", Chaos, Solitons \& Fractals 34, 1430-1439 (2007).

[19] J. H. He, Non-perturbative methods for strongly nonlinear problems (dissertation.de-Verlag im Internet $\mathrm{GmbH}$, Berlin 2006).

[20] J. H. He, "Some asymptotic methods for strongly nonlinear equations", Int. J. Mod. Phys. B, 20, 1141-1199 (2006).

[21] A. Beléndez, C. Pascual, S. Gallego, M. Ortuño and C. Neipp, “Application of a modified He's homotopy perturbation method to obtain higher-order approximations of a $x^{1 / 3}$ force nonlinear oscillator", Phys. Lett. A (2007), doi:10.1016/j.physleta.2007.06.042.

[22] H. M. Liu, "Approximate period of nonlinear oscillators with discontinuities by modified Lindstedt-Poincaré method", Chaos, Solitons \& Fractals 23, 577-579 (2005).

[23] T. Özis and A. Yildirim, “A comparative Study of He's homotopy perturbation method for determining frequency-amplitude relation of a nonlinear oscillator with discontinuities", Int. J. Non-linear Sci. Numer. Simulation 8 (2), 243-248 (2007).

[24] J. H. He, "Homotopy perturbation method for bifurcation on nonlinear problems", Int. J. Non-linear Sci. Numer. Simulation 6, 207-208 (2005).

[25] J. H. He, "The homotopy perturbation method for nonlinear oscillators with discontinuities", Appl. Math. Comp. 151, 287-292 (2004). 
[26] X. C. Cai, W. Y. Wu and M. S. Li, "Approximate period solution for a kind of nonlinear oscillator by He's perturbation method", Int. J. Non-linear Sci. Numer. Simulation 7 (1), 109-117 (2006).

[27] L. Cveticanin, "Homotopy-perturbation for pure nonlinear differential equation", Chaos, Solitons \& Fractals 30, 1221-1230 (2006).

[28] M. Gorji, D. D. Ganji and S. Soleimani, "New application of He's homotopy perturbation method", Int. J. Non-linear Sci. Numer. Simulation 8 (3), 319-328 (2007).

[29] M. S. H. Chowdhury and I. Hashim, "Solutions of time-dependent Emden-Fowler type equations by homotopy-perturbation method", Phys. Lett. A 368, 305-313 (2007).

[30] J. H. He, "Homotopy perturbation method for solving boundary value problems", Phys. Lett. A 350, 87-88 (2006).

[31] A. Beléndez, A. Hernández, T. Beléndez, E. Fernández, M. L. Álvarez and C. Neipp, "Application of He's homotopy perturbation method to the Duffing-harmonic oscillator", Int. J. Non-linear Sci. Numer. Simulation 8 (1), 79-88 (2007).

[32] A. Beléndez, A. Hernández, T. Beléndez, C. Neipp and A. Márquez, "Application of the homotopy perturbation method to the nonlinear pendulum", Eur. J. Phys. 28, 93-104 (2007).

[33] D. D. Ganji and A. Sadighi, "Application of He's homotopy-perturbation method to nonlinear coupled systems of reaction-diffusion equations", Int. J. Non-linear Sci. Numer. Simulation 7 (4), 411-418 (2006).

[34] A. Siddiqui, R. Mahmood and Q. Ghori, "Thin film flow of a third grade fluid on moving a belt by He's homotopy perturbation method", Int. J. Non-linear Sci. Numer. Simulation 7 (1), 15-26 (2006).

[35] M. Rafei and D. D. Ganji, "Explicit solutions of Helmhotlz equation and fifth-order KdV equation using homotopy perturbation method", Int. J. Non-linear Sci. Numer. Simulation 7 (3), 321-328 (2006).

[36] P. D. Ariel and T. Hayat, "Homotopy perturbation method and axisymmetric flow over a stretching sheet", Int. J. Non-linear Sci. Numer. Simulation 7 (4), 399-406 (2006).

[37] F. Shakeri and M. Dehghan, "Inverse problem of diffusion by He's homotopy perturbation method", Phys. Scr. 75, 551-556 (2007). 
[38] M. Dehghan and F. Shakeri, "Solution of a partial differential equation subject to temperature overspecification by He's homotopy perturbation method", Phys. Scr. 75, 778787 (2007).

[39] M. S. H. Chowdhury and I. Hashim, "Solutions of a class of singular second-order IVPs by homotopy-perturbation method", Phys. Lett. A 365, 439-447 (2007).

[40] M. S. H. Chowdhury and I. Hashim, "Application of homotopy-perturbation method to nonlinear population dynamics models", Phys. Lett. A 368, 251-258 (2007).

[41] B. S. Wu, W. P. Sun and C. W. Lim, "An analytical approximate technique for a class of strongly non-linear oscillators", Int. J. Non-linear Mech. 41, 766-774 (2006). 


\section{Figure captions}

Figure 1.- Comparison of the approximate first-order periodic normalized solution (dashed line and circles) with the exact solution (continuous line).

Figure 2.- Comparison of the approximate second-order periodic normalized solution (dashed line and circles) with the exact solution (continuous line).

Figure 3.- Comparison of the approximate third-order periodic normalized solution (dashed line and circles) with the exact solution (continuous line). 
FIGURE 1

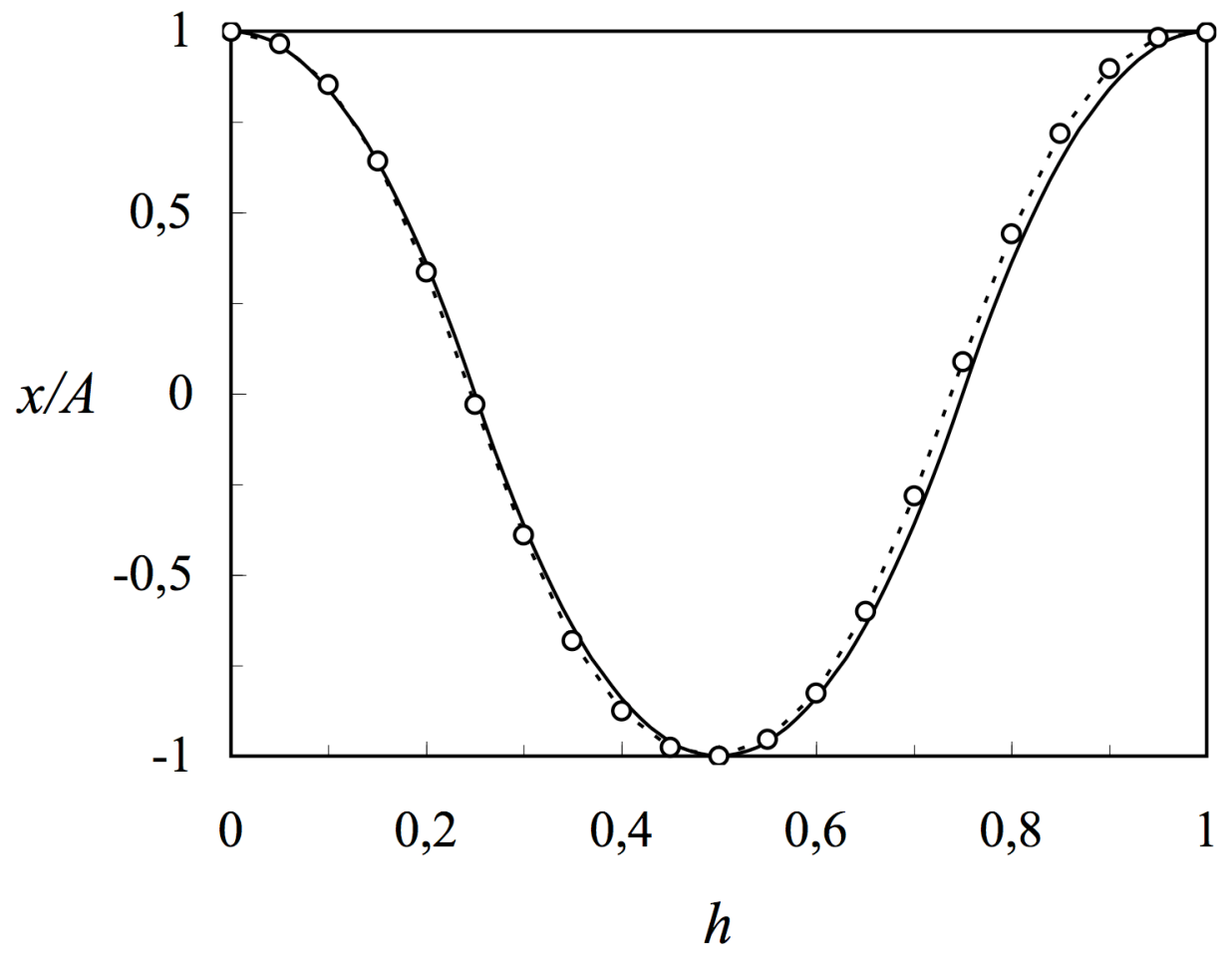


FIGURE 2

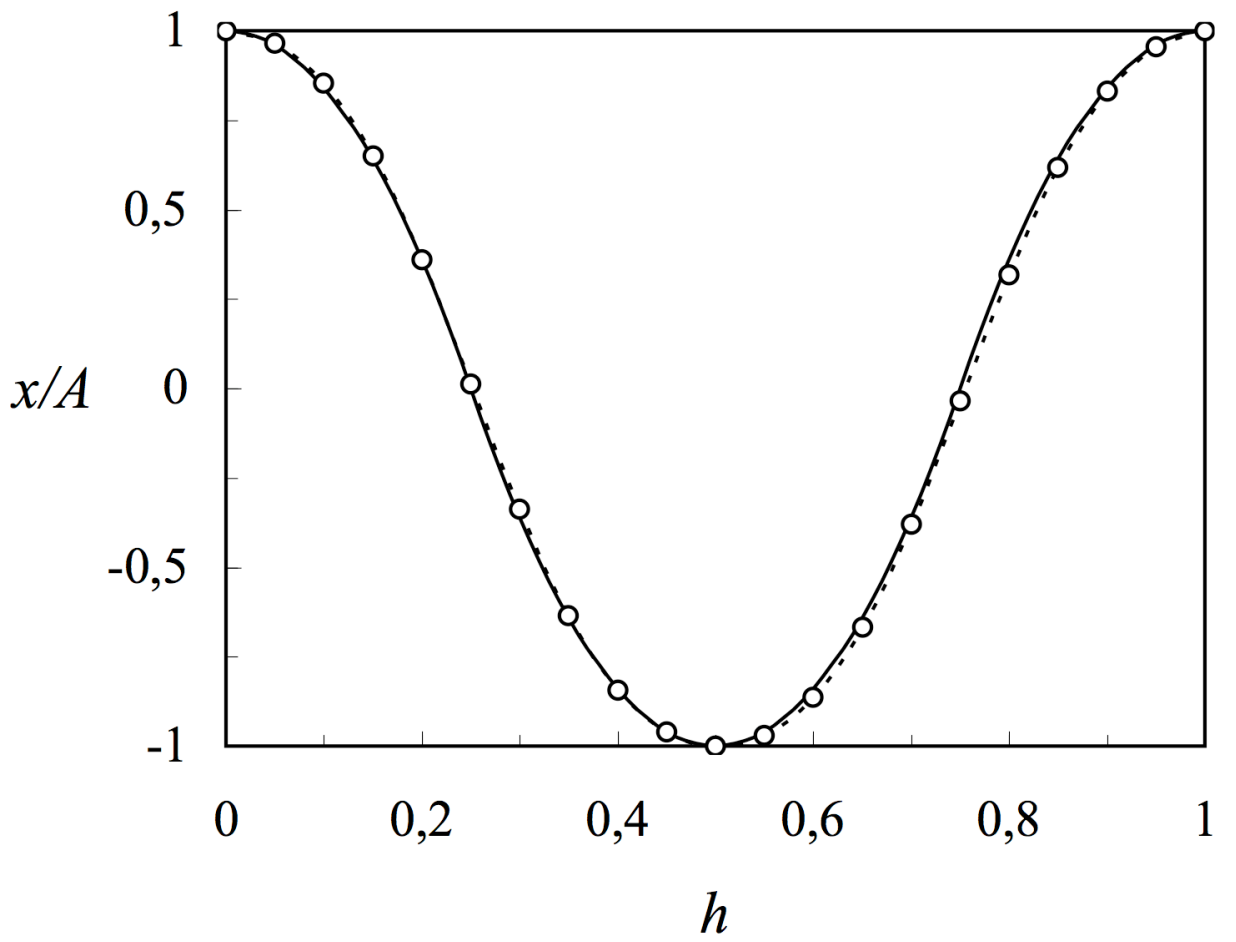


FIGURE 3

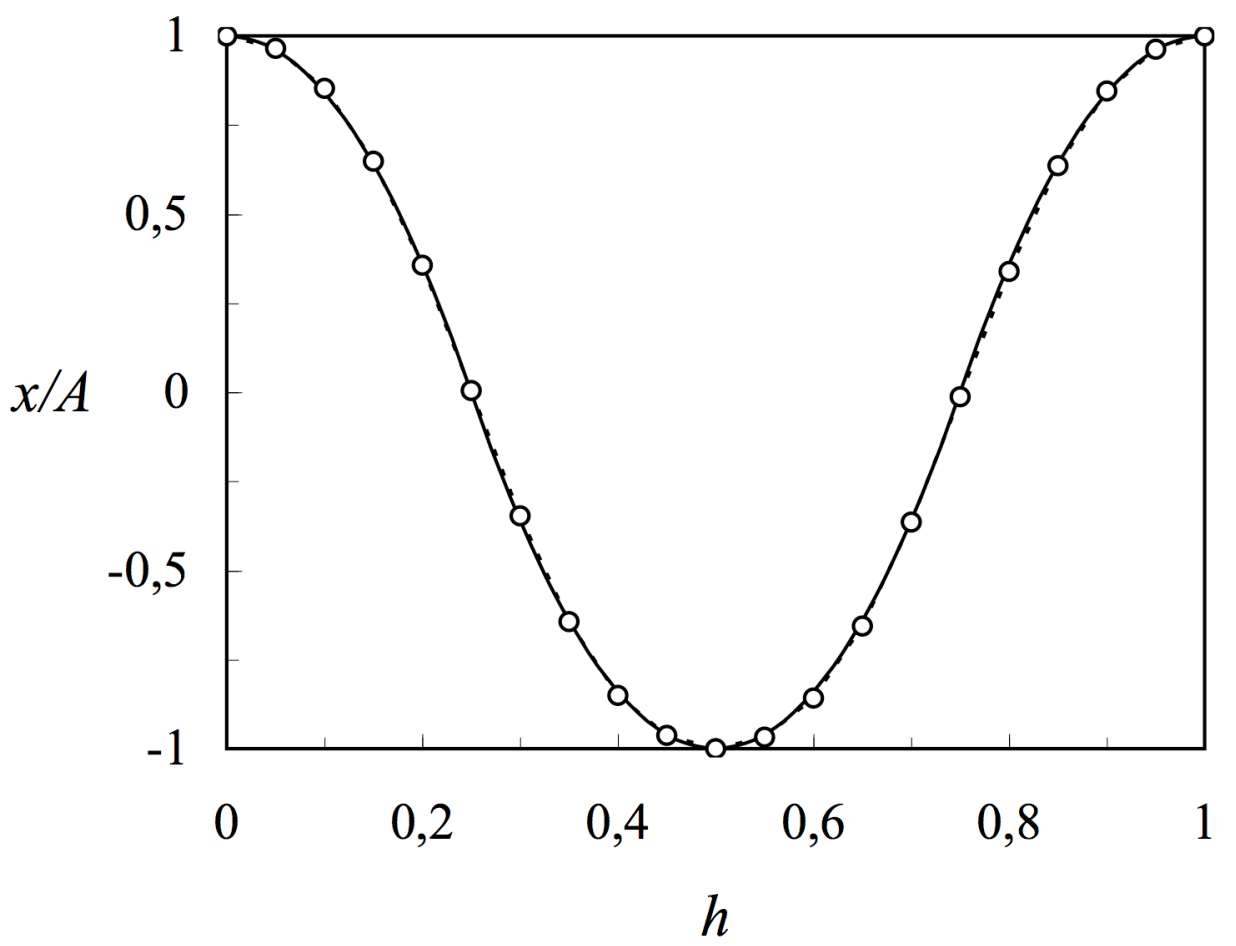

